\title{
Radial fine seek control with fault protection in a digital versatile disc player
}

\author{
Jao-Ming Huang ${ }^{\mathrm{a}, *}$, Jia-Yush Yen ${ }^{\mathrm{b}}$ \\ ${ }^{a}$ Opto-Electronics and Systems Laboratories, Industrial Technology Research Institute, \\ Chutung, Hsinchu 310, Taiwan, ROC \\ ${ }^{\mathrm{b}}$ Department of Mechanical Engineering, National Taiwan University, Taipei, Taiwan 10617, ROC
}

Received 5 May 2003; accepted 30 July 2004

\begin{abstract}
The paper proposes a fine seek servo controller for the digital versatile disk (DVD). The proposed control is an integration of a hybrid track position detector, a position accumulator, a position profile generator, a control finite state machine, an estimator, a defect detector, an RFRP signal compensator, a tracking servo equalizer (for both track-following and fine-seek) and a sled servo equalizer. The integration of these blocks enables a wider range of pull-in area for the servo to switch from track seeking to track following. The implementation results show that the proposed control achieves better performance over the conventional velocity control approach. The experimental results also demonstrated the ability of the control to effectively deal with disks with defects or fingerprints.
\end{abstract}

(C) 2004 Elsevier Ltd. All rights reserved.

Keywords: Digital versatile disk; Servo systems; Track seeking servo; Sensor fusion

\footnotetext{
* Corresponding author.

E-mail addresses: harris_huang@mtk.com.tw (J.-M. Huang), jyen@ntu.edu.tw (J.-Y. Yen).
} 


\section{Introduction}

The optical disk capacity has evolved from 650 Mbytes of the CD-R and CD-RW to 4.7 Gbytes of the digital versatile disk (DVD). To pack so many data in one disk, the data track pitch has shrunk from $1.6 \mu \mathrm{m}$ to $0.74 \mu \mathrm{m}$ on the DVD. The new blue laser DVD would feature track pitch in the range of $0.45 \mu \mathrm{m}$ or even $100 \mathrm{~nm}[1,2]$. The ever decreasing track pitch leads to more frequent track accessing operation, and there is an increasing need to look more closely into the track accessing strategy.

The DVD player typically uses a compound actuator to achieve precision data track positioning (less than $1 / 10$ of the track width) over very large distances (around $33 \mathrm{~mm}$ ). Because the sled actuator is able to travel the entire data region, previous approach had mainly been using the sled actuator for the accessing and use the fine actuator to lock on to the target track. Now the sled actuators are often made of very inexpensive components with friction and backlash. It is very difficult to achieve any precision control. More recent approach had instead uses the sled to move the pick up head to a close neighborhood of the target track and leave it to the fine actuator to seek to the correct target. Basically the range for the fine actuator (about $2.5 \mathrm{~mm}$ ) can cover more than 1000 tracks in the CD-R applications (usually 300 tracks are used for separating short and long seek). For DVD the number would easily exceed 3000. Therefore, newer optical disks have used stepper motor for the sled actuator and leave most of the accessing process to the fine actuator. Recent researches on the DVD seek control were focused on coarse-seek [3-7]; however, the reduction of the seek time is limited by the player mechanisms. With the current increased density of optical discs, like DVD, Blue Ray Disc, or Advance Optical Disc (AOD), the pull-in range which is a range capable of obtaining an effective tracking error (TE) signal at the time of pull-in also becomes narrow. As a result the frequency of overshoots at servo close when switching from fine seeks to track following increases. If the overshoot is out of the pull-in range where the track-following servo loop can take over, the fine-seek may fail or it may take very long for stabilization. Accordingly, the fine-seek control becomes more important. In addition, the authors' experiences showed that the difficulty in track accessing lies more in the difficulty to obtain good position information [8]. The velocity control loop is rendered ineffective by the wrong position feedback information. It is therefore more important to obtain good and adequate position feedback for a decent accessing control. By "good" the authors mean that the position information should be correct. By "adequate" the authors mean that the information needs to be timely.

In this paper, a new fine-seek control scheme is proposed. The controller includes an RFRP signal compensator, a hybrid track position detector for better on-time position information, a modified position estimator and defect detector, a position profile generator, and a tracking servo equalizer. The hybrid track position detector provides on-time and more reliable position information across the tracks. The position estimator provides information on the position and velocity relative to the disk tracks. The fault detector is also based on the estimation. A finite state machine (FSM) then controls the action of the controller. The implementation of the proposed control scheme on a DVD system shows that the system is feasible. In a con- 
ventional fine-seek, the moving velocity is largely affected by the external disturbances and this may result in a failure of the track-following servo. The proposed scheme uses the position feedback to accomplish a fine-seek. Thus, the effect caused by external disturbances can be minimized.

\section{The DVD system model}

As shown in Fig. 1, the radial laser spot positioning system of a DVD player is a two-stage actuator mechanism composed of a coarse actuator and a fine actuator which are named as sled motor and tracking voice coil motor (VCM), respectively.

The tracking VCM, which directly drives the objective lens, is mounted on the top of the pickup traversed by the sled motor. Therefore, the position of the objective lens $\left(x_{\mathrm{f}}\right)$ and that of the pickup $\left(x_{\mathrm{c}}\right)$ determines the position of the laser spot. The two actuators have very different characteristics. The bandwidth of the tracking VCM is wide, while its operating range is small. On the other hand, the operating range of the sled motor covers the entire disc radius at the expense of the bandwidth. Owing to the external disturbance like disc eccentricity, the laser spot position $\left(x_{1}\right)$ is also dependent on the disturbance $\left(x_{\mathrm{d}}\right)$. The plant model can then be described as

$$
\begin{aligned}
& m_{\mathrm{c}} \ddot{x}_{\mathrm{c}}+d_{\mathrm{c}} \dot{x}_{\mathrm{c}}=u_{\mathrm{c}}, \\
& m_{\mathrm{f}} \ddot{x}_{\mathrm{f}}=u_{\mathrm{f}}+d_{\mathrm{f}}\left(\dot{x}_{\mathrm{c}}-\dot{x}_{\mathrm{f}}\right)+k_{\mathrm{f}}\left(x_{\mathrm{c}}-x_{\mathrm{f}}\right),
\end{aligned}
$$
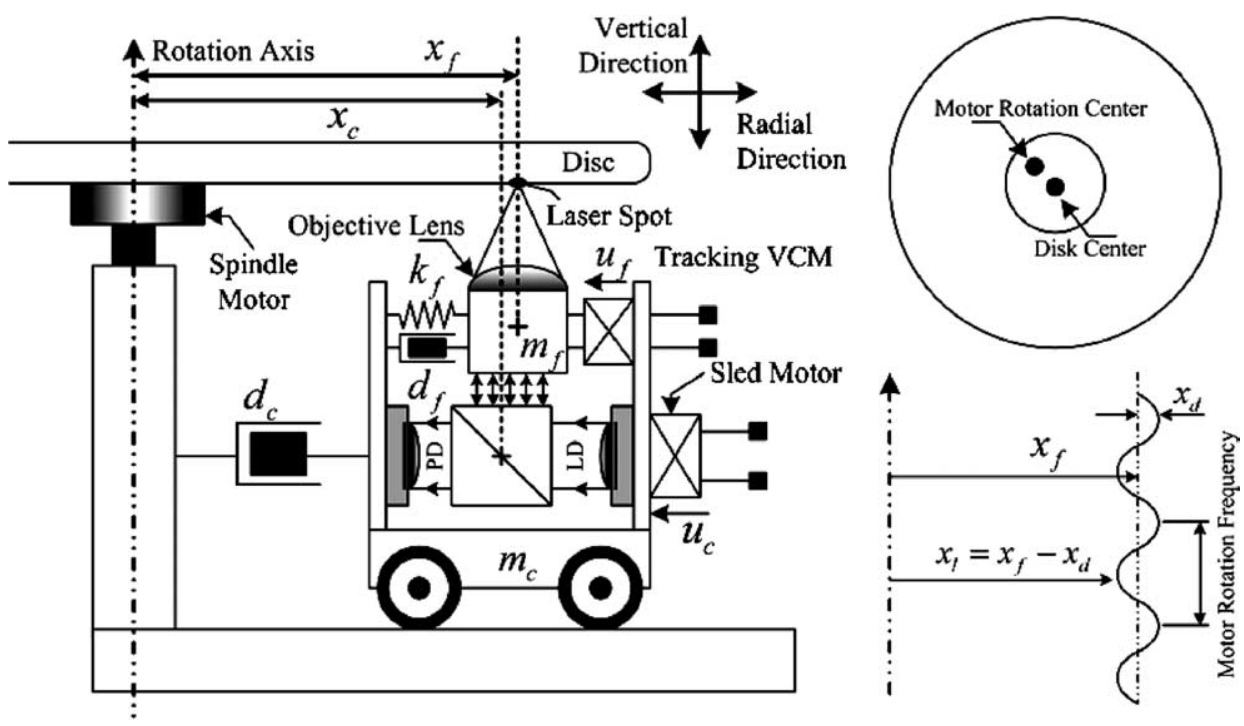

Fig. 1. Model of the radial laser spot positioning system. 


$$
x_{1}=x_{\mathrm{f}}-x_{\mathrm{d}} .
$$

where $m_{\mathrm{c}}$ and $d_{\mathrm{c}}$ are the mass and the viscosity coefficient of the coarse actuator, $m_{\mathrm{f}}$, $d_{\mathrm{f}}$ and $k_{\mathrm{f}}$ are the mass, the viscosity coefficient and the elasticity coefficient of the fine actuator, $u_{\mathrm{c}}$ and $u_{\mathrm{f}}$ are the driving forces from the coarse and the fine actuators, respectively. The effect of fine actuator is neglected in (1) because optical drives often use ball-screws for the sled and the effect of fine actuator become negligible. As shown in Fig. 2, the conventional DVD player uses separate compensated equalizers for the two actuators. The tracking VCM is controlled by the tracking servo equalizer only and the sled servo equalizer is cascaded on top of the tracking servo equalizer. The relative position between the lens and pickup in this case becomes an external disturbance. It is thus desirable that a good fine-seek controller should eliminate the influence caused by the disturbance.

\section{Conventional fine-seek control scheme}

Fig. 2 is the block diagram of the conventional fine seek control scheme using lens kick with velocity control [9-12].

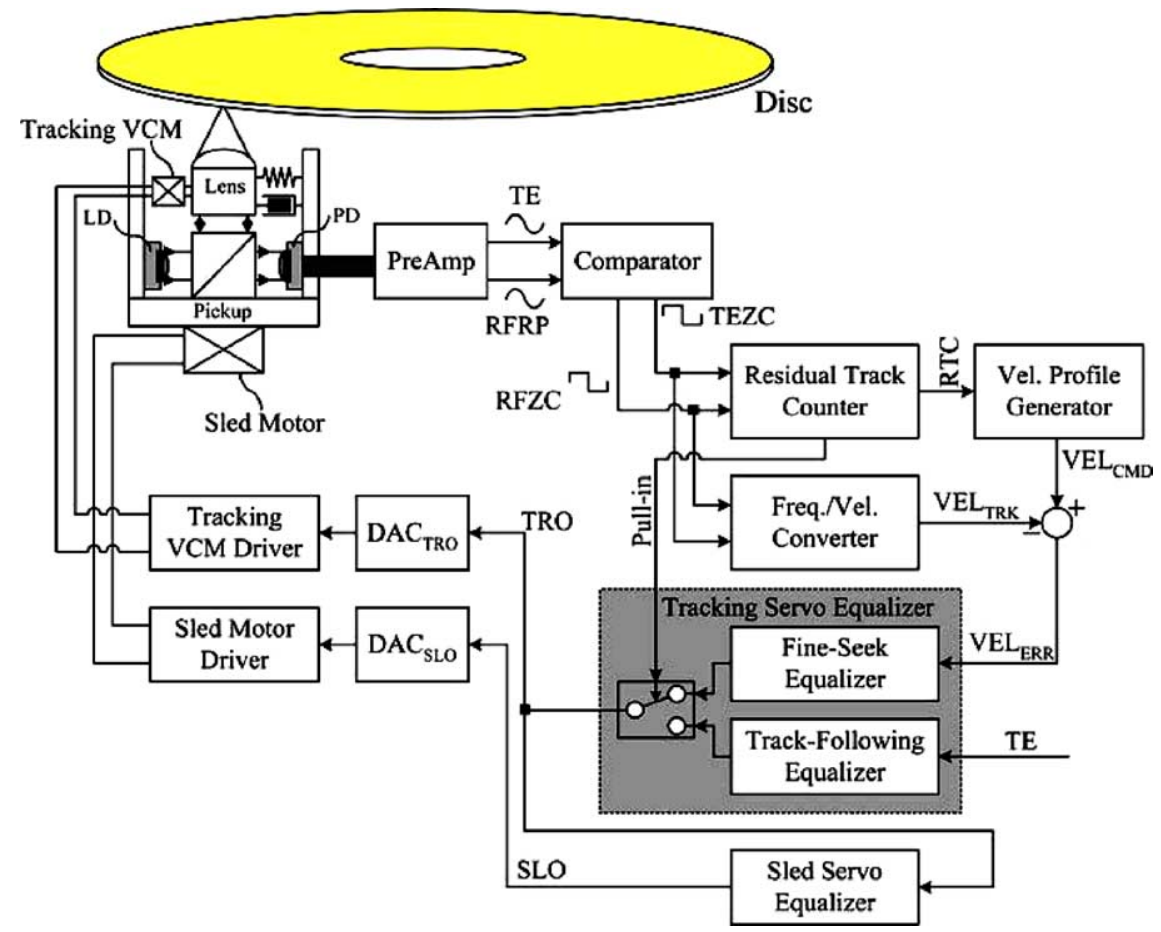

Fig. 2. Block diagram of the conventional fine-seek control scheme. 
The output signals from the photo detector (PD) cells in the pickup are supplied to the PreAmp for generating the tracking error signal and the radio frequency ripple (RFRP) signal. When the laser spot is traversing the disc during a track-seeking operation, tracking error (TE) and the RFRP become modulated relative to the track-cross. The RFRP is a sinusoidal waveform with $90^{\circ}$ phase shift from the TE, which maybe a sinusoidal waveform or a saw tooth waveform depending on the generation method. The TE and the RFRP are both supplied to a comparator circuit to generate the digitized tracking error zero cross (TEZC) signal and digitized RFRP zero cross (RFZC) signal. The residual track counter uses the TEZC and the RFZC as inputs and calculates the residual track count (RTC), which is the number of remaining tracks to the destination. The RTC is then supplied to the velocity profile generator, which uses a look-up table to generate the velocity profile. The velocity profile generator outputs velocity command, $\mathrm{VEL}_{\mathrm{CMD}}$, according to the RTC for the velocity reference. Interested readers please refer to [13] for further detail about the associated circuits. When the RTC becomes zero, the tracking servo system switches to track-following, and the TRO, output of the tracking servo equalizer, is switched to the track-following equalizer, and the laser spot can pull-in to the target track.

The major problem of the conventional fine-seek control scheme is the detection delay time. The frequency-velocity converter for the laser spot velocity measurement calculates an inverse number of the cycles of TEZC and RFZC and converts the inverse number to a relative velocity between the laser spot and disc. Until the next TEZC or RFZC is available, there is no information at all on the velocity measurement. During this time, the control is affected by the latest measurement. As a result, a detection delay occurs. Notice that in reality the velocity of the laser spot varies during the two consecutive measurements. The velocity feedback value may thus be way off. This delay can become worse as the laser spot draws near to the target where the velocity is low. The velocity control is also affected by the external disturbances like disc eccentricity and lens position misalignment. For example, when the real velocity decreases excessively in a low-velocity region right before the pull-in to the track-following operation, the laser spot may actually move in the opposite direction owing to the eccentric velocity from the disc eccentricity. In this case, either the seek time may increase or it may fail all together. Moreover, the detection delay time also results in a steady-state deviation of the final velocity from the desired velocity profile. If the final velocity is out of the range where the track-following servo can take over without failing, the laser spot may not be able to pull in and position itself on the target track.

\section{The fine-seek control scheme}

To deal with the above-mentioned problems, this paper uses a novel fine-seek control based on a hybrid track position (HTP) detector [8]. The HTP detector can measure the relative position between the laser spot and the disc tracks and allows a wide range of linear position measurement. The control based on this signal helps 
to minimize the accessing velocity when pulling in to the destination track. This velocity is the major cause to the overshoot in the transient response of the trackfollowing control [14]. It is also worth mentioning that the moving velocity at the destination track depends on the disc eccentricity and is not controlled by the track-following system whose bandwidth may reach $2.4 \mathrm{kHz}$ for a $1 \mathrm{XS}$ DVD. If the overshoot exceeds the linear range of the TE, then stability is no longer guaranteed. Therefore, the influence of the external disturbance, like disc eccentricity and the lens position misalignment, must be rejected below acceptable level.

Fig. 3 shows the proposed fine seek controller. The control scheme is composed of a HTP detector, a position accumulator, a position profile generator, a control finite state machine (FSM), an estimator, a defect detector, an RFRP compensator, a tracking servo equalizer (for both track-following and fine-seek), a sled servo equalizer and a selector. The HTP detector is a block for detecting a position change of the laser spot relative to the disc. The outputs of HTP detector, HTP and AREA, are supplied to the position accumulator, where HTP is a conversion output of the spot position relative to one track and AREA is an area changeover signal (Fig. 5). By using AREA, the position accumulator in the HTP can detect both the occurrence of track-crossing and the direction of moving. Therefore, the position accumulator

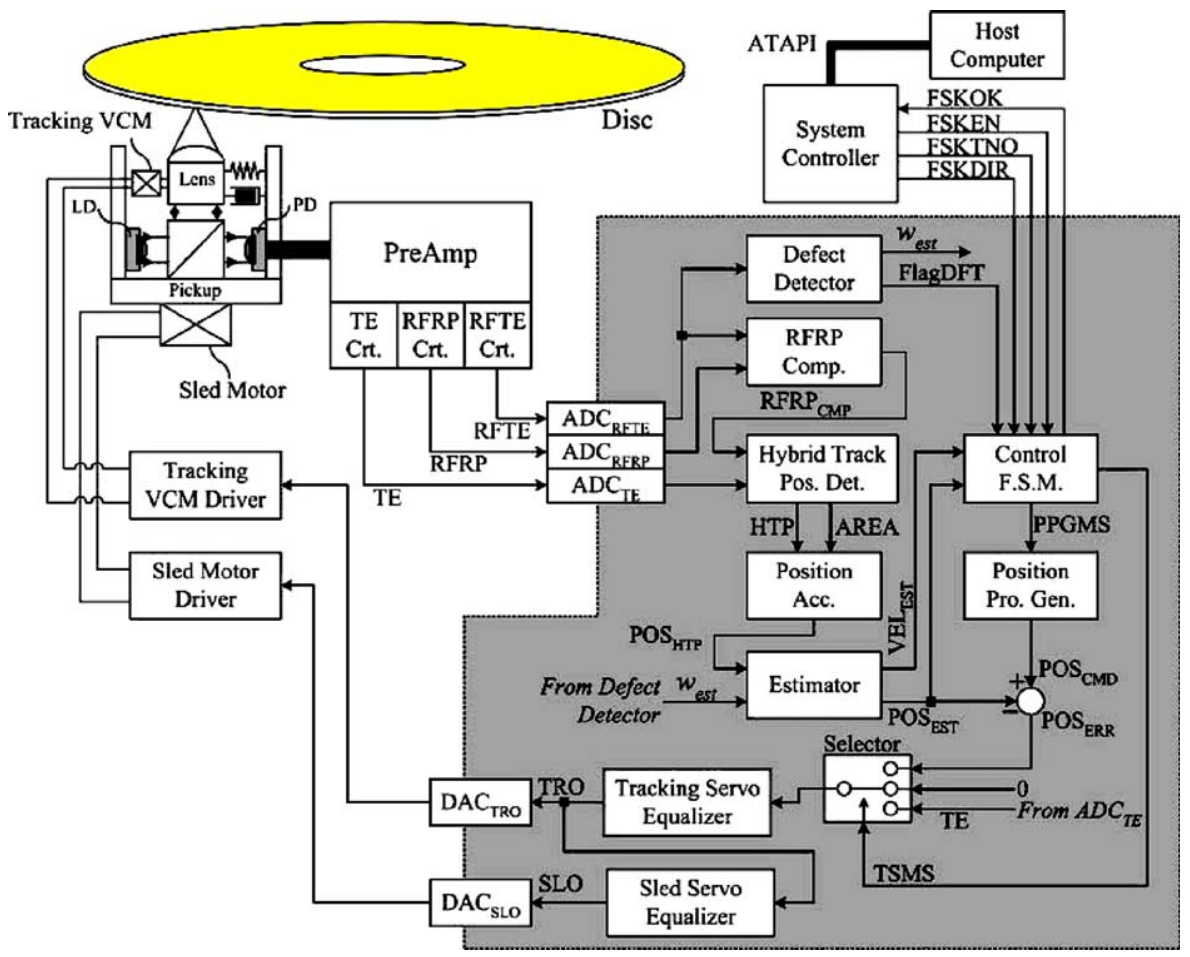

Fig. 3. Block diagram of the fine-seek control scheme. 
can accumulate HTP and count the number of track crossing. The output of the position accumulator, $\mathrm{POS}_{\mathrm{HTP}}$, is fed to the estimator, which is weighted by $w_{\text {est }}$ calculated by the defect detector and will be detailed in following section. The output of the estimator, $\mathrm{POS}_{\mathrm{EST}}$, subtracted from the position command, $\mathrm{POS}_{\mathrm{CMD}}$, then forms the required control feedback error, $\mathrm{POS}_{\mathrm{ERR}}$. The outputs of the estimator, $\mathrm{POS}_{\mathrm{EST}}$ and VEL $\mathrm{EST}_{\mathrm{ET}}$, are also output to the control FSM, which controls the position profile generator. The task of the defect detector is to detect a defect and the output of the defect detector, FlagDFT, will be fed to the control FSM for defect protection. The goal of the RFRP compensator is dynamically to recover the distorted RFRP caused by fingerprints and lens position misalignment. The position profile generator is to generate the position command, POS $_{\mathrm{CMD}}$. The details of the defect detector, RFRP compensator, control FSM, estimator and position profile generator will be introduced in the following sections. The selector is to switch the input of the tracking servo equalizer which is controlled by TSMS outputted by the control FSM.

Fig. 4 is the block diagram of the HTP detector, and Fig. 5 shows the output of the HTP calculator. The combination of the TE $\mathrm{NORM}_{\mathrm{N}}$ and RFRP $\mathrm{NORM}_{\mathrm{N}}$ enables the calculation of a linear HTP signal over a range of two track pitches. This allows for a wider range for seek servo pulling into the track following servo. The readers are

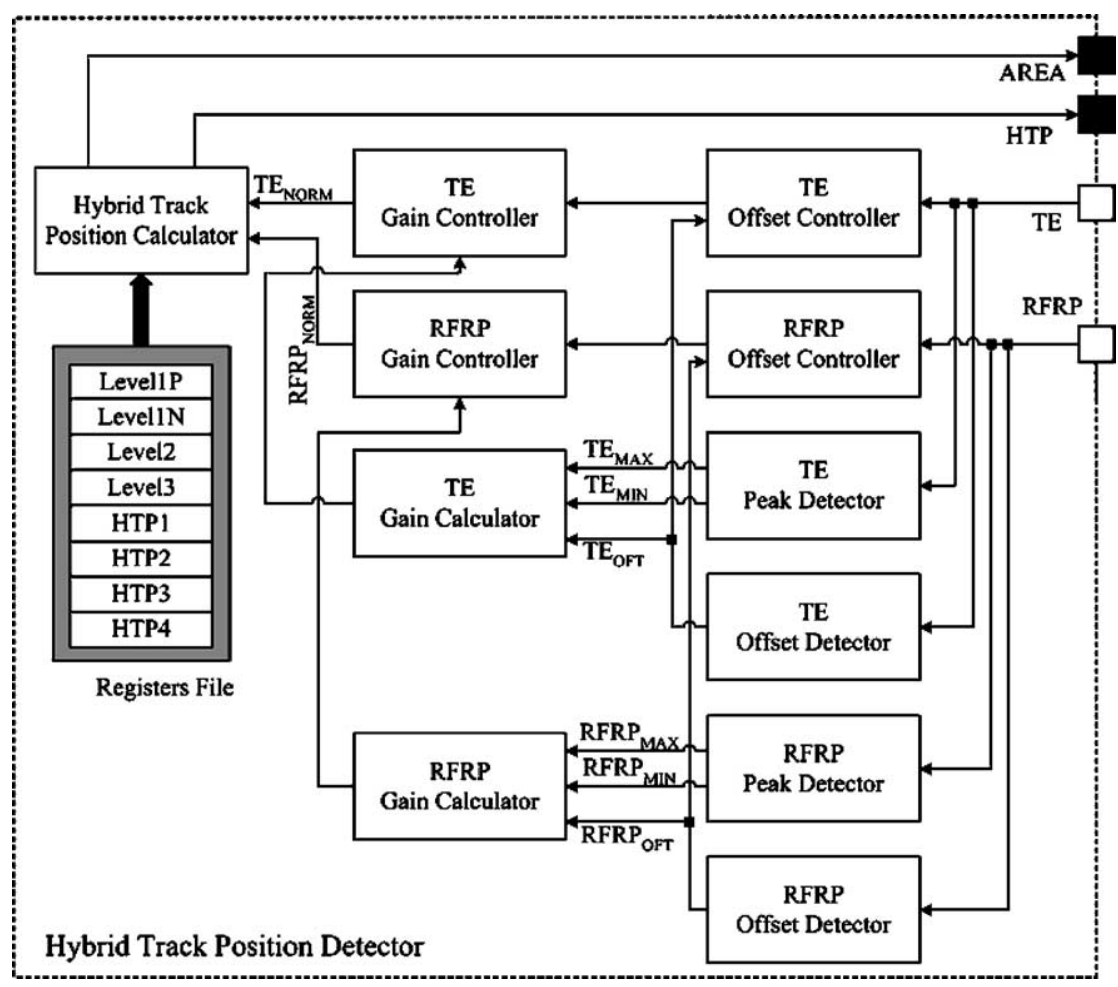

Fig. 4. Block diagram of the hybrid track position detector. 


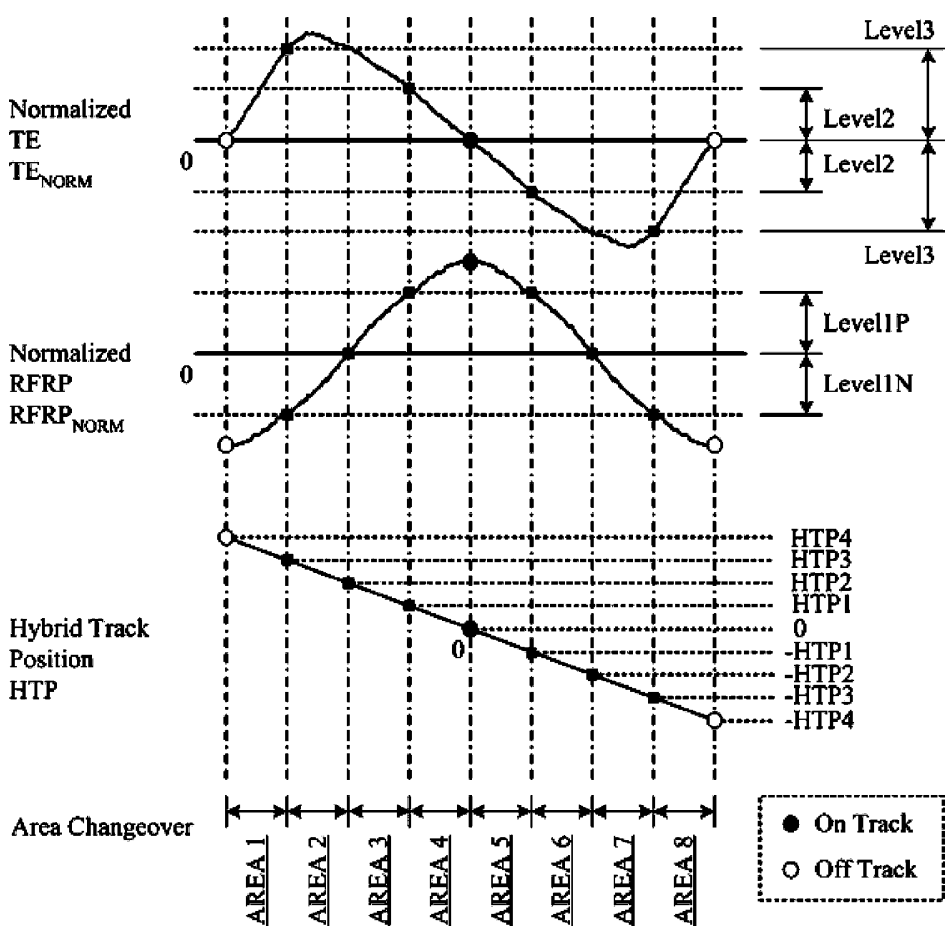

Fig. 5. Principle of the hybrid track position calculator.

referred to [8] for the details of the HTP calculation. After the focusing servo is working stably, TE and RFRP become modulated due to the disc eccentricity. Both TE and RFRP are normalized by adjusting the signal gain and offset. The normalized TE and RFRP, TE NORM $_{\text {and }}$ RFRP $_{\text {NORM }}$, are then supplied to the HTP calculator. The registers file stores the parameters needed in the HTP calculator. Fig. 5 shows an example of the HTP calculator by using DPD TE and RFRP in a case where a relative position between the track and laser spot changes at a predetermined rate. $\mathrm{TE}_{\mathrm{NORM}}$ and $\mathrm{RFRP}_{\mathrm{NORM}}$ corresponding to one track are divided into eight areas. Obviously, in areas 1, 4, 5, 8, TE is linear to HTP, and in areas 2, 3, 6, 7, RFRP is linear HTP. Accordingly, HTP can be generated by using RFRP or TE depending on which signal is linear. The area changeover signal, AREA, is also output to position accumulator, because this signal can be used to detect the direction of the performed track-seeking.

\subsection{RFRP compensator}

From the formation of the position error signal, the RFRP signal from the data channel plays a very important role in the servo control. The RFRP signal is irregularly generated depending on the variations of the quantity of the reflected laser 
light. If there is an irregular output of the RFRP signal, it would be very difficult to determine whether the RFRP signal is correctly focused on a track. Therefore, it is also desirable to have an RFRP compensator that provides a constant RFRP output independent of the laser signal variation.

There are in general three types of RFRP irregularities: lens position misalignment, fingerprint (or rubbish) and defect. If a lens position misalignment occurs during a track-seeking operation, the center level of the RFRP (RFCT) will degrade gradually. This is because the lens position misalignment in the reflection of the laser spot causes the divisions of the PD cell to be illuminated to different degrees even if the reflectivity of a disc remains the same. In this case, the dynamics of the RFCT degradation is slow and the ripple waveform of the RFRP signal will not be distorted. As shown in Fig. 6, which is a 255 tracks of fine-seek over a disc with $150 \mu \mathrm{m}$ eccentricity, during the fine-seek operation, a lens position misalignment and the resulting RFCT degradation, which may be observed from the RFRP plot, was triggered due to the difference between the bandwidth of the dual actuators. However, if a fingerprint (or rubbish) that adheres on a disc is present, the reflectivity of a disc weakens in a wide range and the RFCT will degrade. In this case, the dynamics of the RFCT degradation is fast and the ripple waveform of the RFRP signal may be distorted, as shown in Fig. 7. The proposed RFRP compensator is designated to restore the RFRP signal caused by lens position misalignment and fingerprint.

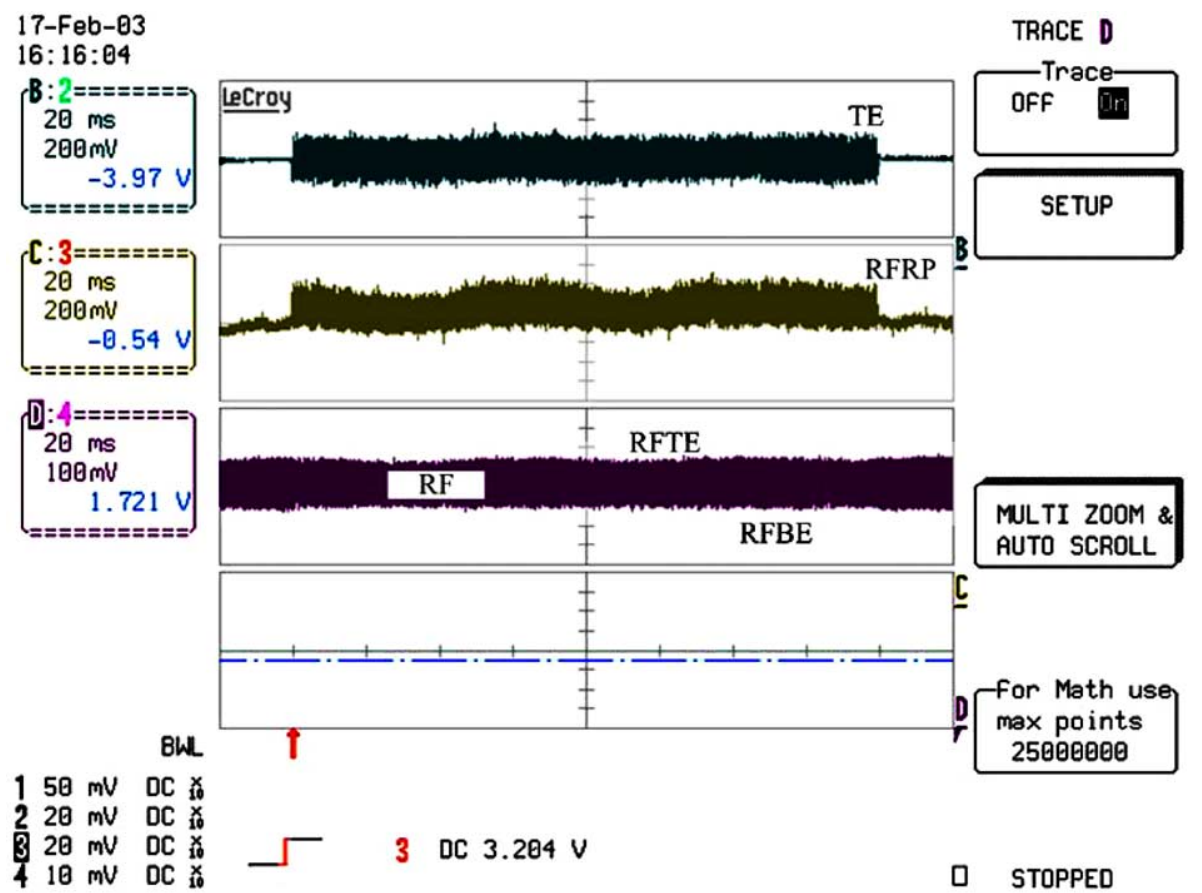

Fig. 6. RFRP distortion caused by lens position misalignment. 


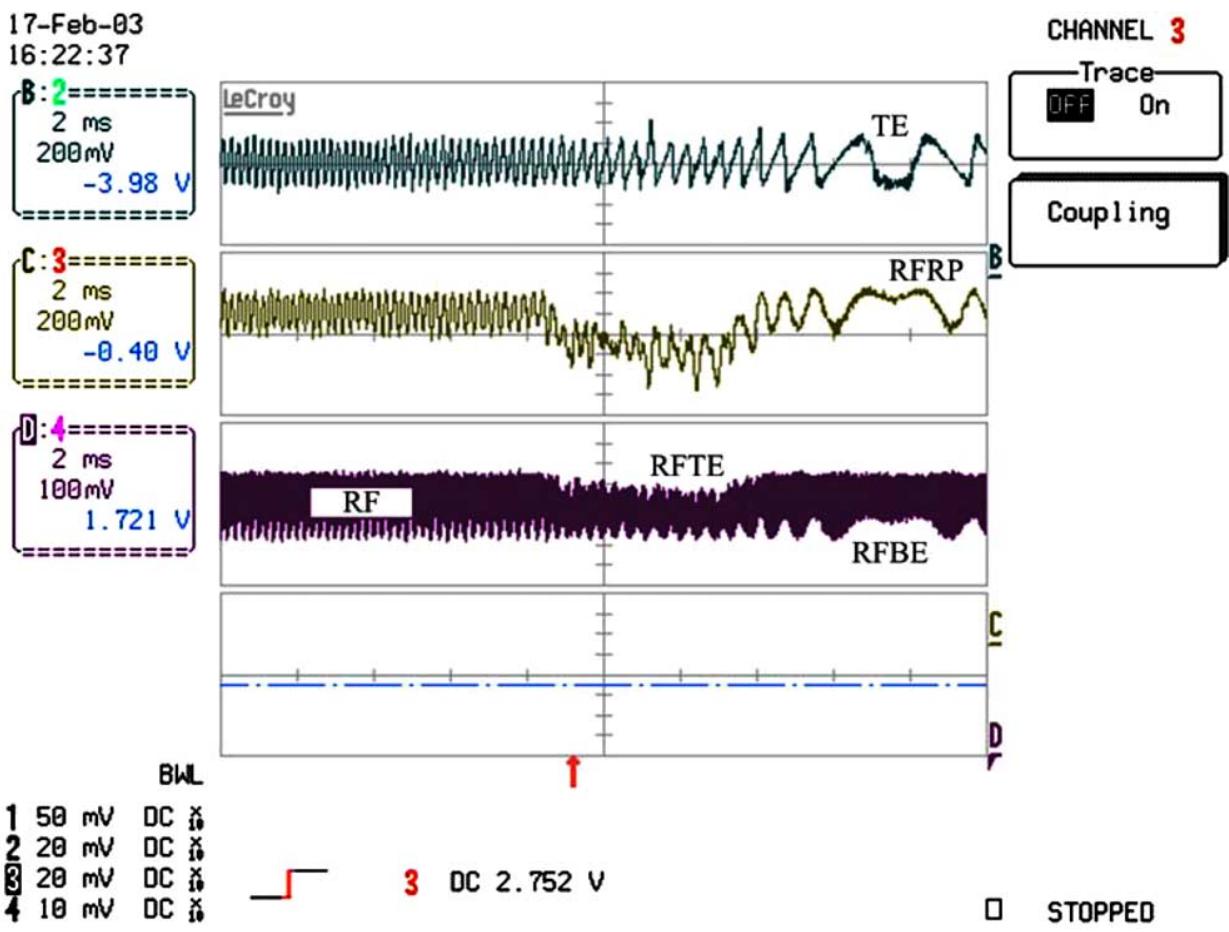

Fig. 7. RFRP distortion caused by a fingerprint.

Unlike fingerprint (or rubbish) and lens position misalignment, a defect will result in a sharp decrease of both the RFRP signal and TE signal in a short-time period because the reflected laser beam is not available at all. In this case, the distorted RFRP signal cannot be restored anymore. To handle defects the fine seek controller would require a fault protection instead of signal compensation. The defect protection will be detailed in the following sections.

Most available research results [15-19] about the compensation of the RFRP signal used the symmetric error of the RFRP signal. By integrating the symmetric error, the drifted RFRP signal can be dynamically compensated. However, because the bandwidth of the compensation loop is limited by the integrator, the previous researches can only reproduce the distorted RFRP signal caused by a lens position misalignment. However, because the distorted RFRP caused by a fingerprint may cover a wide range in bandwidth including the frequency of a track-cross, the dynamics of the previous apparatus is too slow to recovery this type of distortion. The proposed RFRP compensator utilizes the RF signal, which is the source of the RFRP signal, to correct the RFCT. As shown in Fig. 8, if a lens position misalignment or a fingerprint (or a rubbish) occurs, both the RF top envelope (RFTE) and the RFCT are affected. 




Fig. 8. Principle of the RFRP compensator.

Based on this phenomenon, the degradation ratio of the RFTE and RFCT can be calculated as

$$
\begin{aligned}
\mathrm{DR}_{\mathrm{RFTE}} & =\frac{\mathrm{RFTE}_{\mathrm{OK}}-\mathrm{RFTE}}{\mathrm{RFTE}_{\mathrm{OK}}-\mathrm{V}_{\mathrm{REF1}}} \\
\mathrm{DR}_{\mathrm{RFCT}} & =\frac{\mathrm{RFCT}_{\mathrm{OK}}-\mathrm{RFCT}}{\mathrm{RFCT}_{\mathrm{OK}}-\mathrm{V}_{\mathrm{REF} 2}}
\end{aligned}
$$

where $\mathrm{DR}_{\mathrm{RFTE}}$ and $\mathrm{DR}_{\mathrm{RFCT}}$ are the degradation ratio of RFTE and RFCT, respectively, RFTE OK $_{\text {and }}$ RFCT $_{\mathrm{OK}}$ are the RFTE and RFCT level without any lens position alignment, fingerprint or defect. The value of $\mathrm{RFCT}_{\mathrm{OK}}$ and $\mathrm{RFTE}_{\mathrm{OK}}$ can be calculated by an initialization procedure before the track-following control is enabled. $V_{R E F 1}$ is the reference voltage of the RF generation circuit. $V_{R E F 2}$ is the reference voltage of the RFRP generation circuit. However, because the bandwidth of the RF signal and RFRP signal are different, the two signals should be processed by different circuits, which mean different reference voltages and different gains. Accordingly, the degradation ratio between the RFTE and RFCT should be associated with

$$
\mathrm{DR}_{\mathrm{RFCT}}=\mathrm{K} \times \mathrm{DR}_{\mathrm{RFTE}}
$$

By using Eqs. (4)-(6), the RFCT can be dynamically corrected by using

$$
\mathrm{RFCT}=\mathrm{RFCT}_{\mathrm{OK}}-\mathrm{K} \times \frac{\left(\mathrm{RFTE}_{\mathrm{OK}}-\mathrm{RFTE}\right) \times\left(\mathrm{RFCT}_{\mathrm{OK}}-\mathrm{V}_{\mathrm{REF} 2}\right)}{\left(\mathrm{RFTE}_{\mathrm{OK}}-\mathrm{V}_{\mathrm{REF} 1}\right)}
$$

where the gain between the degradation ratio of RFTE and RFCT, K, should be determined depending on the circuit in the PreAmp. The compensated RFRP, RFRP $_{\mathrm{CMP}}$, can be obtained by subtracted from the calculated RFCT

$$
\mathrm{RFRP}_{\mathrm{CMP}}=\mathrm{RFRP}-\mathrm{RFCT}
$$


The performance of the RFRP compensator is also demonstrated in Figs. 6 and 7; the distortion of RFRP caused by lens position misalignment or fingerprint can both be recovered by means of the proposed RFRP compensator.

\subsection{Estimator and defect detector}

The major roll of the estimator in the proposed fine seek controller is to estimate the position and the velocity of the laser spot with respect to the disc tracks. The proposed estimator [20] is formulated as

$$
\begin{aligned}
& \hat{\mathbf{x}}(k+1)=\boldsymbol{\Phi} \hat{\mathbf{x}}(k)+\boldsymbol{\Gamma} \mathbf{u}(k)+\mathbf{L} w_{\text {est }}[\mathbf{y}(k)-\hat{\mathbf{y}}(k)] \\
& \hat{\mathbf{y}}(k)=\mathbf{H} \hat{\mathbf{x}}(k)
\end{aligned}
$$

The estimation is based on the current estimated state of the plant $\boldsymbol{\Phi} \hat{\mathbf{x}}(k)+\Gamma \mathbf{u}(k)$ plus a feedback of the difference between the measured output $\mathbf{y}(k)$ of the plant and the estimated output $\hat{\mathbf{y}}(k)$ multiplied by a fixed feedback gain, $\mathbf{L}$ and by the time varying weight, $w_{\text {est }}$, which is controlled by the defect detector. Referring to Fig. 3, the measured output $\mathbf{y}(k)$ is $\operatorname{POS}_{\mathrm{HTP}}$ and the estimated output $\hat{\mathbf{y}}(k)$ is $\mathrm{POS}_{\mathrm{EST}}$. Eqs. (11)(13) shows how the measurements can be weighted by the factor $w_{\text {est }}$

$$
w_{\text {est }}= \begin{cases}1 & \text { for RFTE } \geqslant \mathrm{RFTE}_{\mathrm{TRD} 1} \\ {\left[\frac{\left(\mathrm{RFTE}_{\mathrm{T}}-\mathrm{RFTE}_{\mathrm{TRD} 2}\right)}{\left(\mathrm{RFTE}_{\mathrm{TRD} 1}-\mathrm{RFTE}_{\mathrm{TRD} 2}\right)}\right]^{\alpha}} & \text { for RFTE } \mathrm{RRD}_{\mathrm{TR}}>\mathrm{RFTE}_{0} \geqslant \mathrm{RFTE}_{\mathrm{TRD} 2} \\ 0 & \text { for RFTE }<\mathrm{RFTE}_{\mathrm{TRD} 2},\end{cases}
$$

$$
\begin{aligned}
& \mathrm{RFTE}_{\mathrm{TRD} 1}=\mathrm{DR}_{\mathrm{TRD} 1} \cdot \mathrm{RFTE}_{\mathrm{OK}}, \\
& \mathrm{RFTE}_{\mathrm{TRD} 2}=\mathrm{DR}_{\mathrm{TRD} 2} \cdot \mathrm{RFTE}_{\mathrm{OK}},
\end{aligned}
$$

where $\mathrm{DR}_{\mathrm{TRD} 1}$ and $\mathrm{DR}_{\mathrm{TRD} 2}$ are two pre-determined thresholds of degradation ratio with respect to $\mathrm{RFTE}_{\mathrm{OK}}$. $\mathrm{RFTE}_{\mathrm{TRD} 1}$ and $\mathrm{RFTE}_{\mathrm{TRD} 2}$ are two thresholds calculated by $\mathrm{DR}_{\mathrm{TRD} 1}$, $\mathrm{DR}_{\mathrm{TRD} 2}$ and $\mathrm{RFTE}_{\mathrm{OK}}$. If the retrieved $\mathrm{RFTE}$ is larger than RFTE $_{T R D 1}$, the track-cross pattern of RFRP will not be really disturbed by the poor reflectivity of a disc and the distortion of RFCT can be recovered by the proposed RFRP compensator. Accordingly, the estimator is fully weighted, $w_{\text {est }}=1$. In the case of a heavy fingerprint, which means RFTE is retrieved between RFTE TRD1 $_{1}$ and $\mathrm{RFTE}_{\mathrm{TRD} 2}$, the track-cross pattern of RFRP will be disturbed and cannot be recovered by RFRP compensator only. At this moment, the estimator is weighted by an exponential function where the exponent $\alpha$ is chosen empirically. When the RFTE is smaller than RFTE $_{\text {TRD2 }}$, the track-cross pattern will be fully destroyed. The estimator is not weighted at all because the real defect of a disc is too severe to retrieve a valid measurement. In this case, the defect detector will output a flag, FlagDFT, to the control FSM. Generally, the value of $\mathrm{DR}_{\mathrm{TRD} 1}$ and $\mathrm{DR}_{\mathrm{TRD} 2}$ are determined by measuring the RFTE and RFRP signals. Furthermore, the value of $\mathrm{DR}_{\mathrm{TRD} 1}$ and $\mathrm{DR}_{\mathrm{TRD} 2}$ are dependent on the characteristics of pickup and PreAmp. 


\subsection{Control FSM and position profile generator}

The control FSM is the kernel of the fine-seek controller proposed in this paper.

Fig. 9 shows a state flow diagram of the control FSM and Table 1 is the description and outputs control of each state, where TSMS is tracking servo mode selection signal, PPGMS is a position profile generator mode selection signal, and FSKOK is fine-seek OK signal. Table 2 shows the transitions of the control FSM, where FSKEN and FlagDFT are external transitions; FlagVMAX, FlagBRK and FlagPIN are internal transitions. Fig. 10 shows how the internal transitions are generated.

Generally, the control FSM works like most fine-seek controllers, including an acceleration state, a constant velocity state, a deceleration state and a pull-in state. The present states are controlled by some internal transition flags, FlagPIN, FlagBRK and FlagVMAX, where the FlagPIN is decided by using

$$
\mid \text { POS }_{\mathrm{EST}}-\text { POS }_{\mathrm{TOL}} \mid \leqslant 1 / 4 \text { track, }
$$

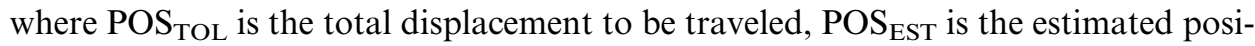
tion output. Because all calculations are performed by a fixed point DSP, sometimes



Fig. 9. State flow of the control finite state machine. 
Table 1

States and outputs of the control finite state machine

\begin{tabular}{lllll}
\hline State & Description & Output control & & \\
\cline { 3 - 5 } & & TSMS & PPGMS & FSKOK \\
\hline 0 & Pull-in state & TE & 0 & 1 \\
1 & Initialization state & TE & 1 & 1 \\
2 & Acceleration state & POS & 2 & 0 \\
3 & Constant velocity state & POS $_{\text {ERR }}$ & 3 & 0 \\
4 & Deceleration state & POS $_{\text {ERR }}$ & 4 & 0 \\
5 & Defect state & 0 & 5 & 0 \\
6 & Recovery state & $\operatorname{POS}_{\text {ERR }}$ & 6 & 0 \\
\hline
\end{tabular}

Table 2

Transitions of the control finite state machine

\begin{tabular}{lll}
\hline Transition & Description & Source \\
\hline FSKEN & Fine-seek enable signal & System controller \\
FlagDFT & Defect flag & Defect detector \\
FlagVMAX & Max velocity flag & Control FSM \\
FlagBRK & Brake flag & Control FSM \\
FlagPIN & Pull-in flag & Control FSM \\
\hline
\end{tabular}

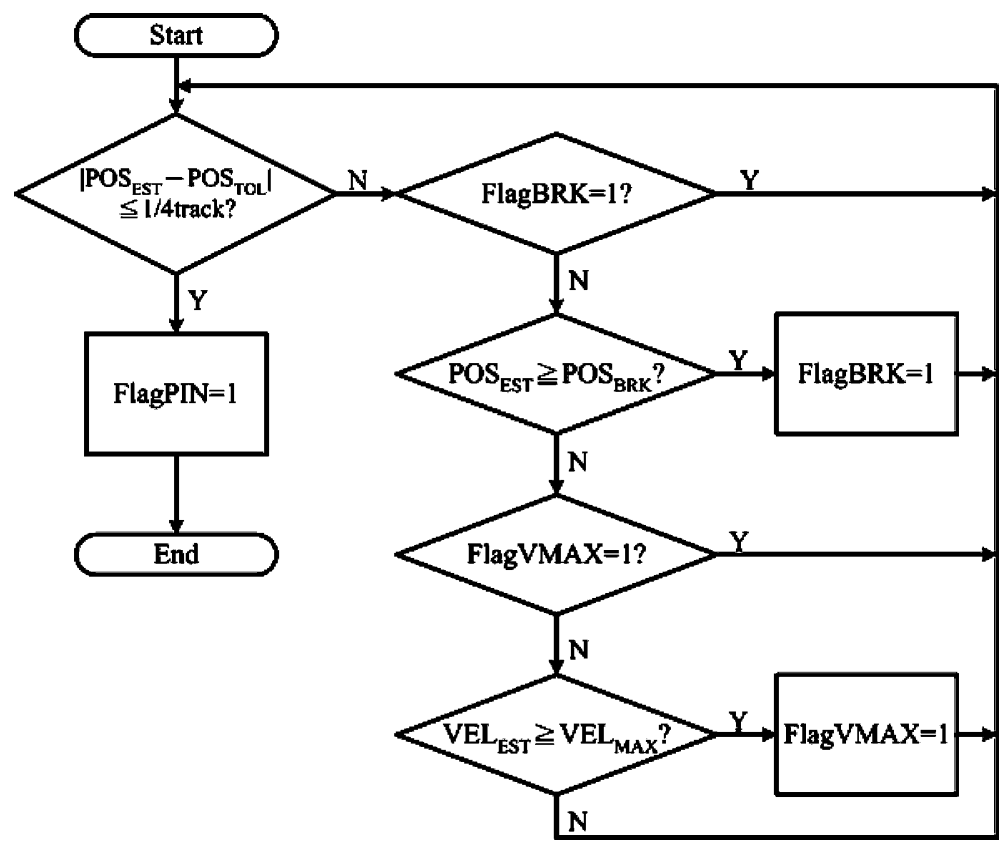

Fig. 10. Internal transitions generation in the control finite state machine. 
it is not possible to exactly move the laser spot to the target position, $\mathrm{POS}_{\mathrm{TOL}}$. Accordingly, when the laser spot position reaches 1/4 track around the target, the FlagPIN is set. Similarly, the FlagBRK is determined by comparing the POS $_{\mathrm{EST}}$ and $\mathrm{POS}_{\mathrm{BRK}}$, where the $\mathrm{POS}_{\mathrm{BRK}}$ is the brake position and calculated by using

$$
\mathrm{POS}_{\mathrm{BRK}}=\operatorname{POS}_{\mathrm{TOL}}-\frac{\mathrm{VEL}_{\mathrm{EST}}^{2}(k)}{2 \cdot \mathrm{DEC}_{\mathrm{CMD}}},
$$

where $\mathrm{DEC}_{\mathrm{CMD}}$ is the desired deceleration value. When $\mathrm{POS}_{\mathrm{EST}}$ is larger than POS $_{\mathrm{BRK}}$, the FlagBRK is set. The FlagVMAX is determined by comparing VEL $\mathrm{EST}_{\mathrm{E}}$ and VEL $\mathrm{VAX}_{\mathrm{MAX}}$, where the VEL $\mathrm{EST}_{\text {is }}$ the estimated velocity and VEL $\mathrm{VAX}_{\mathrm{MA}}$ is the maximum velocity that the HTP detector can measured.

The most special thing of the proposed control FSM is the defect handling. The control FSM includes two states for the defect handling, defect state and recovery state. The defect state is transited by the FlagDFT, which is output from the defect detector. When a defect is detected, the input of the selector in Fig. 3 is switched to 0 for protection. Unlike the defect protection of track-following operation [21] which holding the control output of the tracking VCM before the defect until the defect is passed, the selector input is forced to 0 to prevent a continuous acceleration which may cause an extra-high moving velocity exceeding the bandwidth of the HTP detector or a continuous deceleration which may result in a opposite direction seek. The control FSM also provides a recovery state following the defect state. This is because the error between the estimator and the real output needs some time to converge. After the recovery state, the control FSM can determine the next state according the internal transitions in Fig. 10.

The control FSM also outputs the PPGMS to the position profile generator. The control of the position profile generator is depicted in Table 3. The most important thing in position profile generator is that the $V L_{C M D}$ is held in defect state and recovery state. This can provide a more stable process when a defect is detected. However, during a defect, both the estimator and the accumulation of the position command are kept on working, the position error caused by a defect can be minimized.

Table 3

Control of the position profile generator

\begin{tabular}{ll}
\hline PPGMS & Control of position profile generation \\
\hline 0 & $\operatorname{VEL}_{\mathrm{CMD}}(k+1)=0, \operatorname{VEL}_{\mathrm{CMD}}(k)=0$ \\
& $\operatorname{POS}_{\mathrm{CMD}}(k+1)=0, \operatorname{POS}_{\mathrm{CMD}}(k)=0$ \\
& Setup ACC \\
& output from the system controller \\
2 & $\operatorname{VEL}_{\mathrm{CMD}}(k+1)=\operatorname{VEL}_{\mathrm{CMD}}(k)+\operatorname{ACC}_{\mathrm{CMD}}$ \\
3 & $\operatorname{VEL}_{\mathrm{CMD}}(k+1)=\operatorname{VEL}_{\mathrm{MAX}}$ \\
4 & $\operatorname{VEL}_{\mathrm{CMD}}(k+1)=\operatorname{VEL}_{\mathrm{CMD}}(k)-\operatorname{DEC}_{\mathrm{CMD}}$ \\
5,6 & $\operatorname{VEL}_{\mathrm{CMD}}(k+1)=\operatorname{VEL}_{\mathrm{CMD}}(k)$ \\
$2,3,4,5,6$ & $\operatorname{POS}_{\mathrm{CMD}}(k+1)=\operatorname{POS}_{\mathrm{CMD}}(k)+\left[\operatorname{VEL}_{\mathrm{CMD}}(k+1)+\operatorname{VEL}_{\mathrm{CMD}}(k)\right] / 2$ \\
\hline
\end{tabular}




\subsection{System configuration and tracking servo equalizer design}

Fig. 3 depicts the block diagram of the proposed digital control system. The main processor in the experiment is a single-core 32-bit microcontroller-DSP type of Infineon TriCore, which is optimized for real-time embedded system. Sensor signals are filtered to remove high frequency noise and converted to digital value by on-chip 10-bit A/D converters. Control inputs calculated by DSP are converted by on-chip 10-bit D/A converters. Moreover, the sampling rate of the proposed fine-seek scheme is $100 \mathrm{kHz}$ so that the max detectable velocity of HTP detector is limited to 35 tracks/ms. Besides, both TE and RFRP are filtered by low pass filters for eliminating the high frequency noise, so the bandwidth of TE and RFRP are also restricted within $40 \mathrm{kHz}$. Accordingly, for stable operation of the proposed scheme, the max detectable velocity is set to $35 \mathrm{track} / \mathrm{ms}$ and the max velocity command should be limited within 30 track/ms.

Based on the concept of the fine-seek scheme proposed in this paper, the equalizer of the fine-seek can share the same controller as the track-following servo loop as shown in Fig. 3. This is because both the fine-seek loop and the track-following loop are position feedback loops. Accordingly, the equalizer design should follow the instruction of DVD book ("DVD Specification for Read-Only Disc [22]"), the bandwidth of open loop transfer function (a series connection of compensator and plant) should be kept above $2.4 \mathrm{kHz}$ for 1 XS DVD. Actually, the rotation speed of the disc proposed in this paper is CAV (constant angular velocity) $2 \mathrm{XS}$, so the bandwidth of open loop transfer function should be even higher than $2.4 \mathrm{kHz}$ experimentally. As a result, if the sampling rate of the compensator is $100 \mathrm{kHz}$, the coefficients of a leadlag type equalizer are chosen to be

$$
C(z)=2^{6} \times 0.1456 \times \frac{1-0.9484 z^{-1}-0.9987 z^{-2}+0.9498 z^{-3}}{1-1.2280 z^{-1}+0.1294 z^{-2}+0.1002 z^{-3}} .
$$

The frequency response of the equalizer is shown in Fig. 11. The phase of the leadlag equalizer at $3 \mathrm{kHz}$ is about $60^{\circ}$ that provides a large phase margin for the close loop system.

\section{Experimental results}

Fig. 12 shows the experimental results of five continuous 255 tracks of fine-seeks. The test runs on an ABEX TDR-813 DVD-ROM test disc with $150 \mu \mathrm{m}$ eccentricity. Fig. 12(a), (c) and (e) are the resultant signals from the proposed HTP control scheme. Fig. 12(b) and (d) are the results from the conventional lens kick scheme using velocity control. The experimental data are also compared in Table 4. It is clearly seen that the performance of the proposed HTP control scheme is superior to that of the conventional servo. The proposed HTP control scheme provides shorter seek time, more efficient velocity profile, better reliability, more accurate final velocity control, and more stable pull-in condition. The curves in the experiment are to compare the control ability against the external disturbances, like disc eccentricity 
Tracking Servo Equalizer


Fig. 11. Frequency response of the tracking servo equalizer.
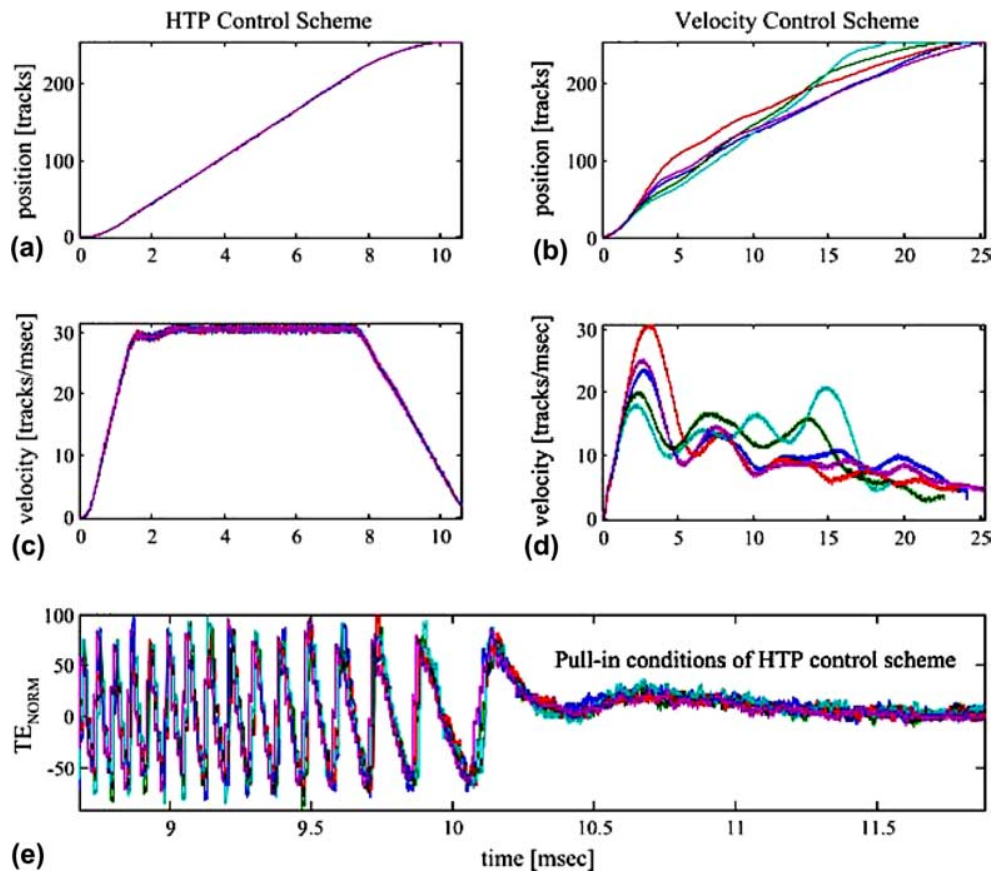

Fig. 12. Experimental results of 255 tracks of fine-seek. 
Table 4

Experimental data of 255 tracks of fine-seek

\begin{tabular}{lll}
\hline Item & HTP control & Velocity control \\
\hline Max. seek time & $10.5637[\mathrm{~ms}]$ & $25.2920[\mathrm{~ms}]$ \\
Min. seek time & $10.5581[\mathrm{~ms}]$ & $19.1400[\mathrm{~ms}]$ \\
Avg. seek time & $10.5604[\mathrm{~ms}]$ & $23.0056[\mathrm{~ms}]$ \\
Max. peak velocity & $31.5059[$ tracks $/ \mathrm{ms}]$ & $30.7588[$ tracks $/ \mathrm{ms}]$ \\
Min. peak velocity & $31.3100[$ tracks $/ \mathrm{ms}]$ & $20.2027[$ tracks $/ \mathrm{ms}]$ \\
Max. final velocity & $2.0493[$ tracks $/ \mathrm{ms}]$ & $5.8127[$ tracks $/ \mathrm{ms}]$ \\
Min. final velocity & $1.9279[$ tracks $/ \mathrm{ms}]$ & $3.0227[$ tracks $/ \mathrm{ms}]$ \\
\hline
\end{tabular}

and lens position misalignment. The conventional lens kick using velocity control is strongly affected by the external disturbance, and the final velocity cannot always converge to the pull-in range of track-following servo loop. Notice that we always design a proximate time optimal velocity profile under rugged consideration. Therefore, a more efficient velocity profile leads to better seek time and a more reliable system.

Fig. 13 and Table 5 show the experimental result of five continuous 7 tracks of fine-seeks. The test disc is also ABEX TDR-813 DVD-ROM. Fig. 13(a), (c) and (e) are the resultant signals from the proposed HTP control scheme. Fig. 13(b)
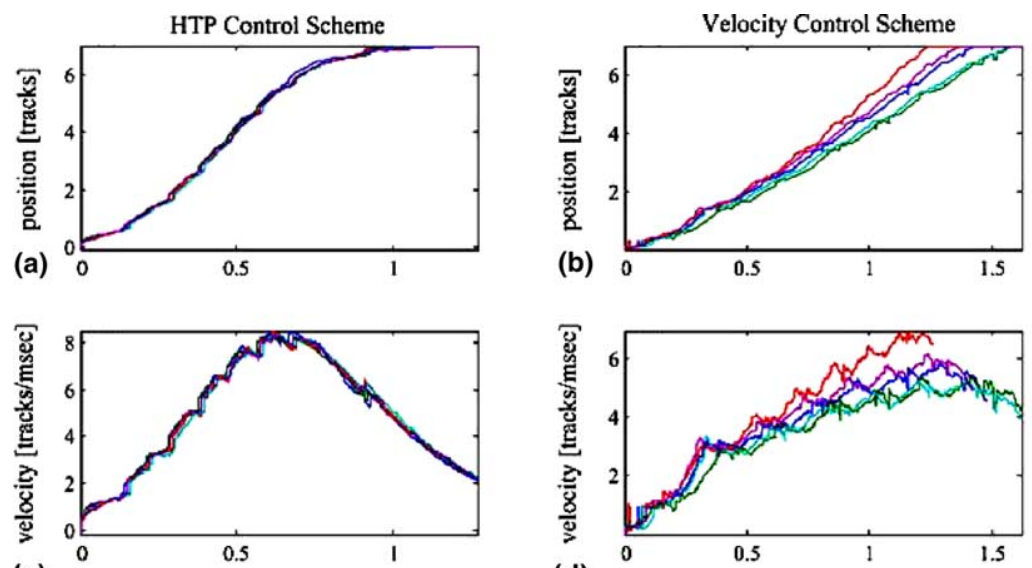

(c)


Fig. 13. Experimental results of seven tracks of fine-seek. 
Table 5

Experimental data of seven tracks of fine track-seeking

\begin{tabular}{lll}
\hline Item & HTP control & Velocity control \\
\hline Max. seek time & $1.2751[\mathrm{~ms}]$ & $1.6200[\mathrm{~ms}]$ \\
Min. seek time & $1.2670[\mathrm{~ms}]$ & $1.2600[\mathrm{~ms}]$ \\
Avg. seek time & $1.2701[\mathrm{~ms}]$ & $1.4802[\mathrm{~ms}]$ \\
Max. peak velocity & $8.5188[$ tracks $/ \mathrm{ms}]$ & $6.9098[\mathrm{tracks} / \mathrm{ms}]$ \\
Min. peak velocity & $8.2937[$ tracks $/ \mathrm{ms}]$ & $5.2341[\mathrm{tracks} / \mathrm{ms}]$ \\
Max. final velocity & $2.2246[$ tracks $/ \mathrm{ms}]$ & $6.4361[\mathrm{tracks} / \mathrm{ms}]$ \\
Min. final velocity & $2.1024[$ tracks $/ \mathrm{ms}]$ & $3.9276[\mathrm{tracks} / \mathrm{ms}]$ \\
\hline
\end{tabular}

and (d) are the results from the conventional lens kick scheme using velocity control. These experiment now show the performance of fine-seek controller against the disc eccentricity when the accessing distance is small. In some application, like DVD video playback, this test is very important. In DVD videodisc, the mpeg2 stream and subtitle are located on different neighboring tracks. The DVD players need to access the mpeg2 stream and the selected subtitle back and forth by continuously repeating the fine-seek operation. If anyone of the repeated fine-seek fails, the video playback will be delayed resulting in frozen pictures. From the curves in Fig. 13 and Table 5, the proposed scheme demonstrates much better velocity control ability and better reliability again.

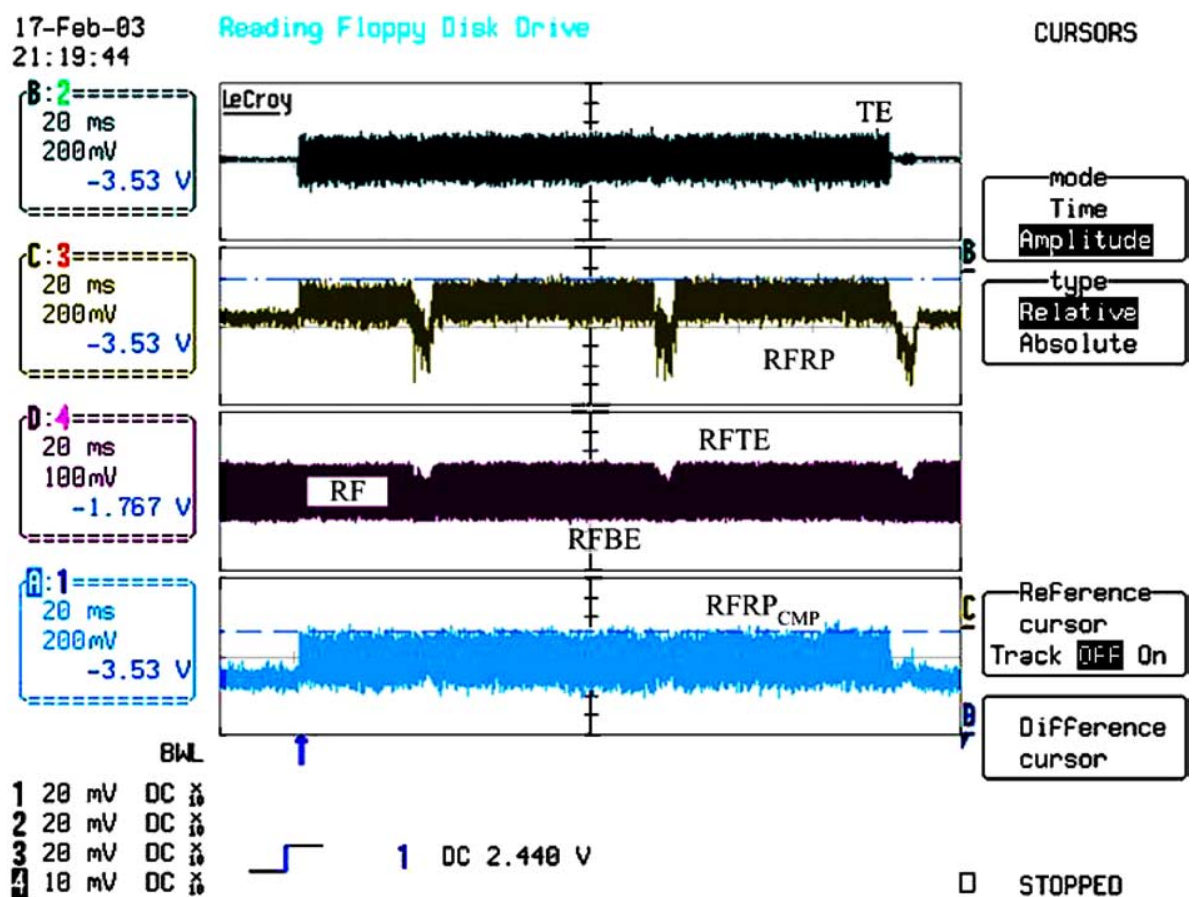

Fig. 14. A 255 tracks of fine-seek when some fingerprints were detected. 


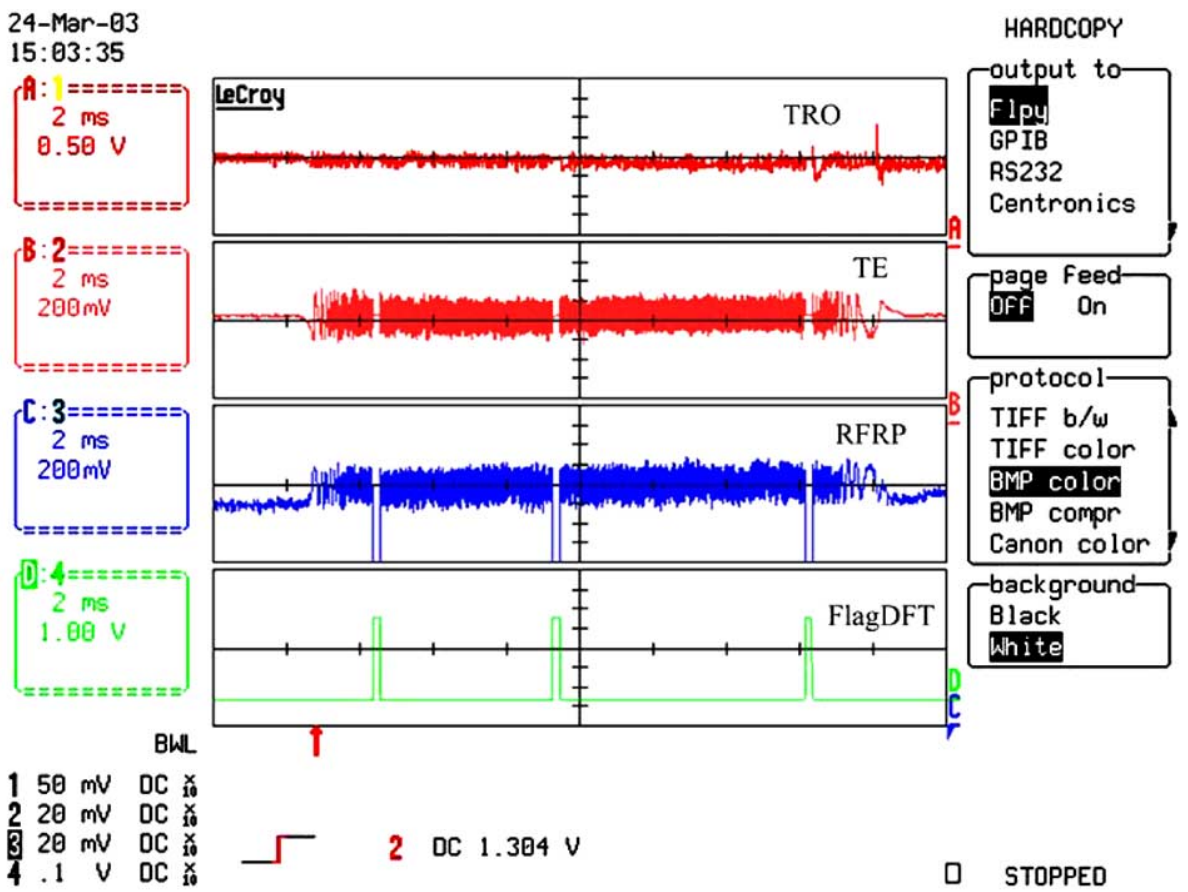

Fig. 15. A 255 tracks of fine-seek when some defects were detected.

Fig. 14 is the experimental result of a 255 tracks of fine-seek when some fingerprints were detected. Because the working of the RFRP compensator, the stability of the fine-seek will not be affected due to the distortion of the RFRP. Fig. 15 is the experimental result of a 255 tracks of fine-seek when some defects were detected. The first defect is detected in the acceleration period, the second defect is in the constant velocity period, and the last defect is in the deceleration period. The proposed control FSM with defect handling shows good protection ability when some defects occurring during a fine-seek.

\section{Conclusions}

This paper proposed a fine-seek control scheme based on the integration of a hybrid track position detector, an RFRP signal compensator, a velocity and position estimator and defect detector, and a position profile generator. The HTP detector and the position accumulator enable a linear position measurement over a wide range of accessing travel. The fine-seek scheme is then designed based upon the HTP position information. Because both the track-following servo and the fine-seek servo are position feedback systems, the two servo loops share the same compensator. The result is a simplified tuning process in the system integration phase. The 
experimental results show that the proposed scheme achieves very accurate position control, better seek time performance, more reliable operations and better velocity control performance. The fine seek experiment also demonstrates the ability of the proposed scheme against the external disturbances, like disc eccentricity and lens position misalignment. The control is effective even when some fingerprints, defects or lens position misalignment occur during a fine-seek.

\section{Acknowledgement}

This work was supported in part by the ROC Government under Grant No. BS123456.

\section{References}

[1] Esener CS, Mark HK, Doyle WD, Keshner M, Mansuripur M, Thompson DA. The future of data storage. WTEC Panel Report. Also Available from <http://www.wtec.org/loyola/hdmem/toc.htm $>$ June, 1999.

[2] Kenya GOTO. Proposal of High bit rate and Tera Bytes Optical Disk Memory (J). The Bull School High-Technol Human Welfare. Tokai University No. 6; 1996. p. 99-112.

[3] McCormick J, Horowitz R. Time optimal seek trajectories for a dual stage optical disk drive actuator. Trans ASME 1991;113:534-6.

[4] Yang JD, Pei XD. Seek time and trajectories of time optimal control for a dual stage optical disk drive actuator. IEEE Trans Magnet 1996;32(5):3857-9.

[5] Yang JD, Pan XD. Time optimal seek control for a coupled dual stage disk drive actuator. IEEE Trans Magnet 1997;33(5):2629-970.

[6] Jin KB, Doh TY, Ryoo JR, Chung MJ. Robust direct seek control for high-speed rotational optical disk drives. IEEE Trans Consumer Electron 1998;44(4):1273-83.

[7] Jin KB, Lee HK, Chung MJ. Direct seek control scheme for high-speed rotational optical disk drives. Electron Lett 1998;34(15):1476-7.

[8] Huang, J-M, Yen JY. A novel track jump controller with hybrid track position detector for the optical disk dirves. In: Proceedings IEEE International Conference on Consumer Electronics, June, Los Angeles, CA; 2003.

[9] Takahara T, Kasahara A. Optical disk tracking system for searching a target track based on a table of compensation reference velocity. US Patent 5566148.

[10] Takahara T, Kasahara A. Optical disc apparatus with accessing using only reference velocity during acceleration and reference and moving velocities during deceleration. US Patent 5497360.

[11] Kao CH, Wang SY. Control device of a pickup head for locating the track position. US 6154424.

[12] Jung RR, Jing KB, Doh TD, Chung MJ. New fine seek control for optical disk drives. In: Proceedings of the Ameriacan Control Conference, vol. 5; 1999. p. 3635-39.

[13] Maeda S. Track jump control apparatus for disc reproducing system. US Patent 5796686.

[14] Sun KCK. Servo system having track crossing detection for an optical disk drive system. US Patent 6320828.

[15] Lai R. Calibration method for slice level of zero cross signal and method of producing track-crossing signal. US Patent Application Publication 2002/0181374 A1.

[16] Armitage PJ, Boddy DI. Apparatus and method providing a mirror averaging function to generate a mirror signal from optical data on an optical disc. US Patent Application Publication 2002/0093904 A1.

[17] Quan TA, Grimsley JL. Dynamic adjustment of disk-drive pickup signals. US Patent 6041028. 
[18] Sun KCK, Grimsley JL. Processing of disc-drive pickup signals. US Patent 6167011.

[19] Yang JH. Apparatus for controlling a mirror signal and a method thereof. US Patent Application Publication 2002/0141314 A1.

[20] Vidal E, Hansen KG, Andersen RS, Poulsen KB, Stoustrup J, Andersen P, Pedersen TS. Linear quadratic controller with fault detection in compact disk player. Proceedings of the IEEE Conference on Control Applications; 2001. p. 77-81.

[21] Ryu T, Yokoyama E, Ogawa M, Kime K. Stable servo system of digital versatile disk player against defects. Jpn Soc Appl Phys 2001;40:5340-1.

[22] DVD specification for read-only disc, version 1.1; Part 1: Physical specifications, Part 2: File system specifications, Part 3: Video specifications; December 1997. DVD Forum. 